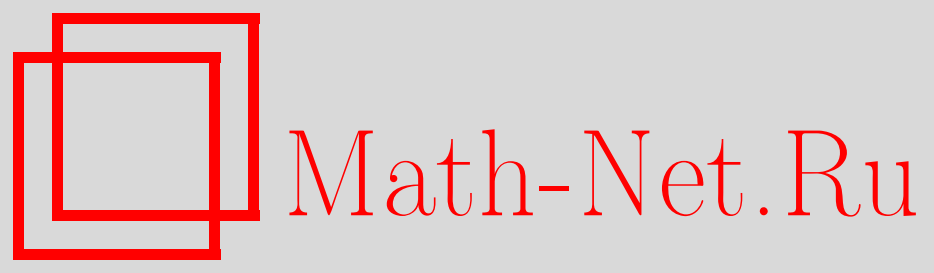

В. Г. Чирский, А. Ю. Нестеренко, Об одном подходе к преобразованию периодических последовательностей, Дискрет. матем., 2015, том 27, выпуск 4, 150-157

DOI: https://doi.org/10.4213/dm1354

Использование Общероссийского математического портала Math-Net.Ru подразумевает, что вы прочитали и согласны с пользовательским соглашением http://www.mathnet.ru/rus/agreement

Параметры загрузки:

IP: 18.234 .156 .22

26 апреля 2023 г., 18:36:27

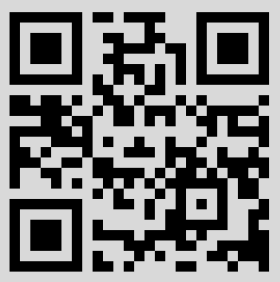




\title{
Об одном подходе к преобразованию периодических последовательностей
}

\author{
() 2015 г. В. Г. Чирский* ${ }^{*}$ А. Ю. Нестеренко**
}

Исследуются арифметические свойства числа $\alpha=\sum_{k=0}^{\infty} \frac{c_{k}}{k !}$ в случае, когда последовательность коэффициентов $\left\{c_{k}\right\}_{k=0}^{\infty}$ является чисто периодической. В качестве приложения доказанных теорем предложено простое преобразование, позволяющее выработать из периодической последовательности $\left\{c_{k}\right\}_{k=0}^{\infty}$ непериодическую последовательность.

Ключевые слова: иррациональность, метод Зигеля-Шидловского, мера линейной независимости, непериодические последовательности.

Одной из важнейших задач при исследовании генераторов случайных чисел является изучение вопроса о периодичности вырабатываемой последовательности. Применение в криптографических приложениях генераторов, вырабатывающих последовательности с малым периодом, может привести к существенному снижению стойкости использующих их приложений, см. [1].

Для усложнения структуры последовательности обычно используются преобразования, называемые фильтрующими или комбинирующими генераторами, см. [1, 3]. Однако за счет преобразования нескольких элементов последовательности в один использование таких генераторов может приводить к уменьшению длины периода вырабатываемой генератором последовательности. В этом случае использование указанных преобразований для изменений свойств периодических последовательностей малой длины является нецелесообразным.

В работе авторами предлагается преобразование, позволяющее получать из периодических последовательностей непериодические последовательности любой длины. Данное преобразование основано на представлении иррациональных чисел в произвольной $b$-ичной системе счисления и использовании вычислений с большим числом знаков.

Пусть $b>1$ - натуральное число и

$$
\alpha=\sum_{k=0}^{\infty} a_{k} b^{k}, \quad \text { где } \quad 0 \leqslant a_{k}<b,
$$

* Место работы: МГУ им. М. В. Ломоносова, e-mail: vgchirskii@yandex.ru

** Место работы: Национальный исследовательский университет «Высшая школа экономики», e-mail: nesterenko_a_y@mail.ru 
- произвольное действительное число. Еще Гауссом было доказано следующее утверждение: для любого натурального числа $b>1$ последовательность чисел $\left\{a_{k}\right\}_{k=0}^{\infty}$ является периодической тогда и только тогда, когда число $\alpha$ рационально, см. [2].

Мы докажем следующую теорему.

Теорема 1. Пусть последовательность цельх чисел $\left\{c_{k}\right\}_{k=0}^{\infty}$ является чисто периодической последовательностью с длиной периода T. Если найдется хотя бы один такой индекс $k$, что $c_{k} \neq 0$, то число

$$
\alpha=\sum_{k=0}^{\infty} \frac{c_{k}}{k !}
$$

является иррачиональным.

Разумеется, эту теорему можно доказать с помощью того же рассуждения, что применяется для доказательства иррациональности числа $e$ (см. теорему 1 [4], стр.51). В этой работе используется классический метод Зигеля-Шидловского в теории трансцендентных чисел, изложенный в монографии [4], с помощью которого удаётся получить количественные оценки, связанные с рассматриваемой задачей.

В силу того, что сумма рационального и отличного от нуля иррационального чисел есть иррациональное число, верно следующее следствие.

Следствие 1. Пусть для последовательности цельх чисел $\left\{c_{k}\right\}_{k=0}^{\infty}$ найдутся такие натуральные числа $k_{0}$ u $T$, что $c_{k}=c_{k+T}$ для всех $k \geqslant k_{0}$. Если найдется хотя бъ один индекс $k \geqslant k_{0}$ такой, что $c_{k} \neq 0$, то число

$$
\alpha=\sum_{k=0}^{k_{0}-1} c_{k} b^{-k}+\sum_{k=0}^{\infty} \frac{c_{k_{0}+k}}{k !}
$$

является иррациональным для любого натурального числа $b$.

Предлагаемое нами преобразование последовательностей заключается в следующем. По последовательности $\left\{c_{k}\right\}$ строится ряд $\sum_{k=0}^{\infty} \frac{c_{k}}{k !}$, сумма которого является иррациональным числом и имеет непериодическое разложение вида (1) в любой позиционной системе счисления. Последовательность коэффициентов этих разложений даёт непериодическую последовательность.

\section{1. Формулировка основного результата}

Для формулировки полученного результата нам потребуется провести некоторые вычисления. Так как последовательность $\left\{c_{k}\right\}_{k=0}^{\infty}$ чисто периодическая с периодом $T$, то для любого индекса $k=0,1,2, \ldots$ имеет место равенство $c_{k+T}=c_{k}$, поэтому

$$
\alpha=\sum_{k=0}^{\infty} \frac{c_{k}}{k !}=\sum_{k=0}^{T-1} c_{k} \sum_{s=0}^{\infty} \frac{1}{(k+s T) !} .
$$


Используя обозначение $(x)_{s}=x(x+1) \cdots(x+s-1)$, определим функции комплексного переменного $z$

$$
\begin{gathered}
f_{0}(z)=\sum_{s=0}^{\infty} \frac{\left(\frac{z}{T}\right)^{T s}}{(1)_{s}\left(\frac{1}{T}\right)_{s} \ldots\left(\frac{T-1}{T}\right)_{s}}, \\
f_{k}(z)=\sum_{s=0}^{\infty} \frac{\left(\frac{z}{T}\right)^{T s}}{(1)_{s}\left(\frac{1}{T}+1\right)_{s} \ldots\left(\frac{k}{T}+1\right)_{s}\left(\frac{k+1}{T}\right)_{s} \ldots\left(\frac{T-1}{T}\right)_{s}}, k=1, \ldots, T-1 .
\end{gathered}
$$

При $k=1, \ldots, T-1$ имеют место равенства

$$
\begin{gathered}
(k+s T) !=1 \cdot 2 \cdot 3 \cdot \ldots \cdot(k+s T)= \\
=1(1+T) \ldots(1+s T) \cdot 2(2+T) \ldots(2+s T) \cdot \ldots \cdot k(k+T) \ldots(k+s T) \times \\
\quad \times(k+1)(k+1+T) \ldots(k+1+(s-1) T) \cdot \ldots \cdot T(2 T) \ldots(s T)= \\
=k ! \cdot s ! \cdot T^{s T}\left(\frac{1}{T}+1\right) \ldots\left(\frac{1}{T}+s\right) \ldots\left(\frac{k}{T}+1\right) \ldots\left(\frac{k}{T}+s\right) \times \\
\times\left(\frac{k+1}{T}\right) \ldots\left(\frac{k+1}{T}+s-1\right) \ldots\left(\frac{T-1}{T}\right) \ldots\left(\frac{T-1}{T}+s-1\right)= \\
=k ! \cdot T^{s T} \cdot(1)_{s} \cdot\left(\frac{1}{T}+1\right)_{s} \ldots \cdot\left(\frac{k}{T}+1\right)_{s}\left(\frac{k+1}{T}\right)_{s} \ldots \cdot\left(\frac{T-1}{T}\right)_{s} .
\end{gathered}
$$

Кроме того, при $k=0$

$$
\begin{aligned}
T^{T s}(1)_{s}\left(\frac{1}{T}\right)_{s} \ldots & \left(\frac{T-1}{T}\right)_{s}==T^{s} \cdot s ! \cdot T^{s}\left(\frac{1}{T}\right) \cdot\left(\frac{1}{T}+1\right) \ldots\left(\frac{1}{T}+s-1\right) \ldots T^{s} \times \\
& \times\left(\frac{T-1}{T}\right) \cdot\left(\frac{T-1}{T}+1\right) \ldots\left(\frac{T-1}{T}+s-1\right)= \\
=T \cdot 2 T \cdot \ldots \cdot s T & \cdot 1(T+1) \ldots(T+(s-1) T) \ldots(T-1)(2 T-1 \ldots(s T-1)=(T s) ! .
\end{aligned}
$$

Следовательно, согласно (5), (6), (7), (8), получаем

$$
\begin{aligned}
\sum_{s=0}^{\infty} \frac{1}{(k+s T) !} & =\sum_{s=0}^{\infty} \frac{1}{k ! \cdot T^{s T} \cdot(1)_{s} \cdot\left(\frac{1}{T}+1\right)_{s} \cdot \ldots \cdot\left(\frac{k}{T}+1\right)_{s}\left(\frac{k+1}{T}\right)_{s} \cdot \ldots \cdot\left(\frac{T-1}{T}\right)_{s}}= \\
= & \frac{1}{k !} \sum_{s=0}^{\infty} \frac{\left(\frac{1}{T}\right)^{T s}}{(1)_{s} \cdot\left(\frac{1}{T}+1\right)_{s} \cdot \ldots \cdot\left(\frac{k}{T}+1\right)_{s}\left(\frac{k+1}{T}\right)_{s} \cdot \ldots \cdot\left(\frac{T-1}{T}\right)_{s}}=\frac{1}{k !} f_{k}(1) .
\end{aligned}
$$

Ввиду (4), (9) получаем

$$
\alpha=\sum_{k=0}^{\infty} \frac{c_{k}}{k !}=\sum_{k=0}^{T-1} c_{k} \sum_{s=0}^{\infty} \frac{1}{(k+s T) !}=\sum_{k=0}^{T-1} \frac{c_{k}}{k !} f_{k}(1) .
$$

Таким образом, для доказательства иррациональности числа $\alpha$ достаточно доказать линейную независимость чисел

$$
1, f_{0}(1), f_{1}(1), \ldots, f_{T-1}(1)
$$


над полем рациональных чисел. Действительно, если $\alpha=\frac{p}{q}-$ рациональное число, то из равенства

$$
-\frac{p}{q} \cdot 1+\sum_{k=0}^{T-1} \frac{c_{k}}{k !} f_{k}(1)=0
$$

сразу следует линейная зависимость чисел (10).

Линейная независимость чисел (10) над полем рациональных чисел является следствием более общей теоремы. Для её формулировки потребуется ещё одно определение. Мерой линейной независимости чисел $\alpha_{1}, \ldots, \alpha_{m}$ называется функция

$$
L\left(\alpha_{1}, \ldots, \alpha_{m}, H\right)=\min \left|h_{1} \alpha_{1}+\ldots+h_{m} \alpha_{m}\right|,
$$

где минимум берётся по всевозможным наборам целых чисел $h_{1}, \ldots, h_{m}$, среди которых есть отличные от нуля, и $H=\max \left(\left|h_{1}\right|, \ldots,\left|h_{m}\right|\right)$.

Теорема 2. Пусть последовательность цельх чисел $\left\{c_{k}\right\}_{k=0}^{\infty}$ является чисто периодической последовательностью с длиной периода Т. Если найдется хотя бъ один такой индекс $k$, что $c_{k} \neq 0$, то для любого $\varepsilon, 0<\varepsilon<\frac{1}{2}$, существует эфбективная постоянная $b>0$, зависящая от чисел $c_{0} \ldots c_{T-1}$ и чисел $T, \varepsilon$,

$$
L\left(1, f_{0}(1), f_{1}(1), \ldots, f_{T-1}(1), H\right)>b H^{1-m-\varepsilon} .
$$

\section{2. Доказательство теоремы 2}

В доказательстве теоремы 2 используется метод Зигеля-Шидловского. Чтобы применить его, докажем сначала, что функции

$$
1, f_{0}(z), f_{1}(z), \ldots, f_{T-1}(z)
$$

линейно независимы над полем рациональных функций $\mathbb{C}(z)$. Это утверждение можно доказать различными способами. Использованный ниже способ, хотя и не самый короткий, даёт наиболее полное представление о структуре рассматриваемого множества функций.

Пусть $S_{1}=\left\{f_{0}(z), \ldots, f_{T-1}(z)\right\}, S_{2}=\left\{h_{0}(z), \ldots, h_{T-1}(z)\right\}$ два набора функций комплексного переменного. Мы будем говорить, что они линейно эквивалентны над полем рациональных функций $\mathbb{C}(z)$ и обозначать $S_{1} \sim S_{2}$, если найдутся такие $a_{i j}(z) \in \mathbb{C}(z)$, что

$$
h_{j}(z)=\sum_{i=0}^{T-1} a_{i j}(z) f_{i}(z), \quad j=0,1, \ldots, T-1
$$

и $\operatorname{det}\left|a_{i j}(z)\right| \neq 0$.

Лемма 1. Имеет место линейная эквивалентность

$$
\left\{f_{0}(z), \ldots, f_{T-1}(z)\right\} \sim\left\{f_{0}(z), \ldots, f_{0}^{(T-1)}(z)\right\} .
$$


Доказательство. Введем оператор $\delta: \mathbb{C}(z) \rightarrow \mathbb{C}(z)$ равенством $\delta=z \frac{d}{d z}$ и докажем равенства

$$
\begin{aligned}
\delta f_{0}(z) & =\frac{z^{T}}{(T-1) !} f_{T-1}(z), \\
(\delta+k) f_{k}(z) & =k f_{k-1}(z), k=1, \ldots, T-1 .
\end{aligned}
$$

Действительно,

$$
\begin{aligned}
& (\delta+k) f_{k}(z)= \\
& =\left(z \frac{d}{d z}+k\right)\left(\sum_{s=0}^{\infty} \frac{\left(\frac{z}{T}\right)^{T s}}{(1)_{s}\left(\frac{1}{T}+1\right)_{s} \ldots\left(\frac{k}{T}+1\right)_{s}\left(\frac{k+1}{T}\right)_{s} \ldots\left(\frac{T-1}{T}\right)_{s}}\right)= \\
& =\sum_{s=0}^{\infty} \frac{(T s+k)\left(\frac{z}{T}\right)^{T s}}{(1)_{s}\left(\frac{1}{T}+1\right)_{s} \ldots\left(\frac{k}{T}+1\right)_{s}\left(\frac{k+1}{T}\right)_{s} \ldots\left(\frac{T-1}{T}\right)_{s}} .
\end{aligned}
$$

Далее, из равенства $\left(\frac{k}{T}+1\right)_{s}=\left(\frac{k}{T}+1\right) \ldots\left(\frac{k}{T}+s\right)$ следует, что

$$
\begin{aligned}
\frac{(T s+k)}{\left(\frac{k}{T}+1\right)_{s}}=\frac{T\left(\frac{k}{T}+s\right)}{\left(\frac{k}{T}+1\right) \ldots\left(\frac{k}{T}+s\right)} & = \\
& =\frac{T}{\left(\frac{k}{T}+1\right) \ldots\left(\frac{k}{T}+s-1\right)}=\frac{k}{\frac{k}{T} \ldots\left(\frac{k}{T}+s-1\right)}=\frac{k}{\left(\frac{k}{T}\right)_{s}} .
\end{aligned}
$$

Таким образом,

$$
(\delta+k) f_{k}(z)=k\left(\sum_{s=0}^{\infty} \frac{\left(\frac{z}{T}\right)^{T s}}{(1)_{s}\left(\frac{1}{T}+1\right)_{s}\left(\frac{k-1}{T}+1\right)_{s}\left(\frac{k}{T}\right)_{s} \ldots\left(\frac{T-1}{T}\right)_{s}}\right)=k f_{k-1}(z),
$$

$k=1, \ldots, T-1$. Так как

$$
f_{0}(z)=\sum_{s=0}^{\infty} \frac{\left(\frac{z}{T}\right)^{T s}}{(1)_{s}\left(\frac{1}{T}\right)_{s} \ldots\left(\frac{T-1}{T}\right)_{s}}=1+\sum_{s=1}^{\infty} \frac{\left(\frac{z}{T}\right)^{T s}}{(1)_{s}\left(\frac{1}{T}\right)_{s} \ldots\left(\frac{T-1}{T}\right)_{s}},
$$

то выполнено равенство

$$
\delta f_{0}(z)=z \frac{d}{d z}\left(1+\sum_{s=1}^{\infty} \frac{\left(\frac{z}{T}\right)^{T s}}{(1)_{s}\left(\frac{1}{T}\right)_{s} \ldots\left(\frac{T-1}{T}\right)_{s}}\right)=\sum_{s=1}^{\infty} \frac{T s\left(\frac{z}{T}\right)^{T s}}{(1)_{s}\left(\frac{1}{T}\right)_{s} \ldots\left(\frac{T-1}{T}\right)_{s}} .
$$

Для любого $s \geqslant 1$ имеем $(1)_{s}=s$ !, поэтому $\frac{s}{(1)_{s}}=\frac{1}{(1)_{s-1}}$ и

$$
\begin{aligned}
\sum_{s=1}^{\infty} \frac{T s\left(\frac{z}{T}\right)^{T s}}{(1)_{s}\left(\frac{1}{T}\right)_{s} \ldots\left(\frac{T-1}{T}\right)_{s}}=T \sum_{s=1}^{\infty} \frac{\left(\frac{z}{T}\right)^{T s}}{(1)_{s-1}\left(\frac{1}{T}\right)_{s} \ldots\left(\frac{T-1}{T}\right)_{s}}= \\
=T \sum_{r=0}^{\infty} \frac{\left(\frac{z}{T}\right)^{T(r+1)}}{(1)_{r}\left(\frac{1}{T}\right)_{r+1} \ldots\left(\frac{T-1}{T}\right)_{r+1}} .
\end{aligned}
$$

По определению, $(a)_{r+1}=a(a+1) \ldots(a+r+1)=a(a+1)_{r}$, следовательно,

$$
\begin{aligned}
\left(\frac{1}{T}\right)_{r+1} \ldots\left(\frac{T-1}{T}\right)_{r+1}=\frac{1}{T}\left(\frac{1}{T}+1\right)_{r} & \ldots \frac{T-1}{T}\left(\frac{T-1}{T}+1\right)_{r}= \\
& =\frac{(T-1) !}{T^{T-1}}\left(\frac{1}{T}+1\right)_{r} \ldots\left(\frac{T-1}{T}+1\right)_{r} .
\end{aligned}
$$


Используя это равенство, получим

$$
\begin{aligned}
\delta f_{0}(z)=T \sum_{r=0}^{\infty} \frac{\left(\frac{z}{T}\right)^{T(r+1)}}{(1)_{r}\left(\frac{1}{T}\right)_{r+1} \ldots\left(\frac{T-1}{T}\right)_{r+1}} & = \\
& =T\left(\frac{z}{T}\right)^{T} \cdot \frac{T^{T-1}}{(T-1) !} f_{T-1}(z)=\frac{z^{T}}{(T-1) !} f_{T-1}(z) .
\end{aligned}
$$

Таким образом, равенства (12) доказаны, а из них следует утверждение леммы:

$$
\left\{f_{0}(z), \ldots, f_{T-1}(z)\right\} \sim\left\{f_{0}(z), \delta f_{0}(z), \ldots, \delta^{T-1} f_{0}(z)\right\} \sim\left\{f_{0}(z), \ldots, f_{0}^{(T-1)}(z)\right\} .
$$

Следствие 2. Функиии $1, f_{0}(z), \ldots, f_{T-1}(z)$ составляют решение системы линейных дифберенииальных уравнений вида

$$
y_{i}^{\prime}=Q_{i}(z)+\sum_{j=0}^{T-1} Q_{i, j}(z) y_{j}, j=0, \ldots, T-1, Q_{i}(z), Q_{i, j}(z) \in \mathbb{C}(z), i, j=0, \ldots, T-1 .
$$

Лемма 2. Выполнено равенство

$$
f_{0}(z)=\frac{1}{T} \sum_{j=0}^{T-1} \exp \left(\zeta^{j} z\right), \quad \text { əде } \quad \zeta=\exp \left(\frac{2 \pi i}{T}\right)
$$

Доказательство. Из равенств (5) и (8) следует, что

$$
f_{0}(z)=\sum_{s=0}^{\infty} \frac{\left(\frac{z}{T}\right)^{T s}}{(1)_{s}\left(\frac{1}{T}\right)_{s} \ldots\left(\frac{T-1}{T}\right)_{s}}=\sum_{s=0}^{\infty} \frac{z^{T s}}{T^{T s}(1)_{s}\left(\frac{1}{T}\right)_{s} \ldots\left(\frac{T-1}{T}\right)_{s}}=\sum_{s=0}^{\infty} \frac{z^{T s}}{(T s) !} .
$$

Далее,

$$
\frac{1}{T} \sum_{j=0}^{T-1} \exp \left(\zeta^{j} z\right)=\frac{1}{T} \sum_{j=0}^{T-1} \sum_{r=0}^{\infty} \frac{\zeta^{j r} z^{r}}{r !}=\frac{1}{T} \sum_{r=0}^{\infty}\left(\sum_{j=0}^{T-1} \zeta^{j r}\right) \frac{z^{r}}{r !}
$$

Так как $\zeta=\exp \left(\frac{2 \pi i}{T}\right)$, то при $T$, делящемся на $r$, сумма $\sum_{j=0}^{T-1} \zeta^{j r}$ равна $T$. Если же $T$ не делится на $r$, то $\sum_{j=0}^{T-1} \zeta^{j r}=\frac{1-\zeta^{j T}}{1-\zeta^{j}}=0$. Таким образом,

$$
\frac{1}{T} \sum_{j=0}^{T-1} \exp \left(\zeta^{j} z\right)=\frac{1}{T} \sum_{s=0}^{\infty} \frac{T z^{T s}}{(T s) !}=f_{0}(z)
$$

Лемма доказана.

Лемма 3. Имеет место линейная эквивалентность

$$
\left\{f_{0}(z), \ldots, f_{T-1}(z)\right\} \sim\left\{\exp \left(\zeta^{j} z\right), \quad j=0,1, \ldots, T-1\right\} .
$$

Доказательство. Из леммы 2 следует, что

$$
f_{0}^{\prime}(z)=\frac{1}{T} \sum_{j=0}^{T-1} \zeta^{j} \exp \left(\zeta^{j} z\right)
$$




$$
\begin{gathered}
f_{0}^{\prime \prime}(z)=\frac{1}{T} \sum_{j=0}^{T-1} \zeta^{2 j} \exp \left(\zeta^{j} z\right), \\
\ldots \\
f_{0}^{(T-1)}(z)=\frac{1}{T} \sum_{j=0}^{T-1} \zeta^{(T-1) j} \exp \left(\zeta^{j} z\right) .
\end{gathered}
$$

Следовательно, матрица перехода от системы $\left\{f_{0}(z), \ldots, f_{T-1}(z)\right\}$ к системе $\left\{\exp \left(\zeta^{j} z\right), \quad j=0,1, \ldots, T-1\right\}-$ невырождена, поскольку ее определитель равен

$$
\operatorname{det}\left|\begin{array}{cccc}
1 & 1 & \ldots & 1 \\
1 & \zeta & \ldots & \zeta^{T-1} \\
1 & \zeta^{2} & \ldots & \zeta^{2(T-1)} \\
\ldots & \ldots & \ldots & \ldots \\
1 & \zeta^{T-1} & \ldots & \zeta^{(T-1)^{2}}
\end{array}\right|
$$

т.е. представляет собой отличный от нуля определитель Вандермонда.

Лемма 4. Если $\alpha_{1}, \ldots, \alpha_{l}$-различные комплексные числа, то фбункции $\exp \left(\alpha_{1} z\right), \ldots, \exp \left(\alpha_{l} z\right)$ линейно независимы над полем $\mathbb{C}(z)$ рациональных функиий.

Предположим противное, и пусть имеют место равенства вида

$$
p_{1}(z) \exp \left(\alpha_{1} z\right)+\ldots+p_{l}(z) \exp \left(\alpha_{l} z\right)=0
$$

с коэффициентами - многочленами от z. Выберем среди таких равенств такое, которое содержит наименьшее количество функций $\exp \left(\alpha_{1} z\right), \ldots, \exp \left(\alpha_{l} z\right)$, скажем, $k \leqslant l$ и, произведя, если потребуется, перенумерацию, предположим, что

$$
p_{1}(z) \exp \left(\alpha_{1} z\right)+\ldots+p_{k}(z) \exp \left(\alpha_{k} z\right)=0 \text {. }
$$

При этом, по определению этого равенства, все $p_{1}(z), \ldots, p_{k}(z)$ отличны от нуля. Следовательно, равенство (13) содержит хотя бы два слагаемых.

Продифференцируем равенство (13) и получим

$$
\left(p_{1}^{\prime}(z)+\alpha_{1} p_{1}(z)\right) \exp \left(\alpha_{1} z\right)+\ldots+\left(p_{k}^{\prime}(z)+\alpha_{k} p_{k}(z)\right) \exp \left(\alpha_{k} z\right)=0 .
$$

Равенства (13) и (14) должны быть пропорциональны друг другу, как линейные формы от $\exp \left(\alpha_{1} z\right), \ldots, \exp \left(\alpha_{k} z\right)$, иначе из них можно было бы исключить одну из функций $\exp \left(\alpha_{1} z\right), \ldots, \exp \left(\alpha_{k} z\right)$, получив при этом равенство, содержащее меньшее, чем равенство (13), количество функций $\exp \left(\alpha_{1} z\right), \ldots, \exp \left(\alpha_{k} z\right)$. Следовательно, должно выполняться соотношение

$$
\frac{p_{1}^{\prime}(z)+\alpha_{1} p_{1}(z)}{p_{1}(z)}=\frac{p_{k}^{\prime}(z)+\alpha_{k} p_{k}(z)}{p_{k}(z)} \text {, или }\left(\ln \frac{p_{1}(z)}{p_{k}(z)}\right)^{\prime}=\alpha_{k}-\alpha_{1},
$$

или

$$
\frac{p_{1}(z)}{p_{k}(z)}=C \exp \left(\left(\alpha_{k}-\alpha_{1}\right) z\right)
$$


что противоречит рациональности функции $\frac{p_{1}(z)}{p_{k}(z)}$.

Лемма 4 вместе с леммами 1 и 3 , даёт утверждение о линейной независимости над полем $\mathbb{C}(z)$ функций $1, f_{0}(z), \ldots, f_{T-1}(z)$. По следствию леммы 1 они составляют решение системы линейных дифференциальных уравнений с коэффициентами из $\mathbb{C}(z)$.

Эти функции являются так называемыми $E$-функциями (см. [4; стр. 184]).

Применяя теорему 1 из [4; стр. 354], получаем утверждение теоремы 2.

Отметим, что эффективность постоянной $b$ следует из доказанной в [5] эффективности постоянной $n_{0}$, см. [4; стр. 106].

Авторы выражают профессору В.Х. Салихову благодарность за полезные обсуждения.

\section{Список литературы}

1. Алферов А.П., Зубов А.Ю., Кузьмин А.С., Черемушкин А.В., Основы криптографии, учебное пособие, Гелиос, Москва, 2001, 480 с.

2. Нестеренко Ю.В., Теория чисел, Академия, Москва, 2008, 272 с.

3. Фомичев В.М., Методъ дискретной математики в криптологии, Диалог-МИФИ, Москва, 2010, 424 с.

4. Шидловский А.Б., Трансцендентные числа, Наука, Москва, 1987, 417 с.

5. Bertrand D., Chirskii V., Yebbou Y., "Effective estimates for global relations on Euler-type series", Ann.Fac.Sci.Toulouse, XIII:2 (2004), 241-260. 